\title{
Analysis of Age Hardening Precipitates of Al-Zn-Mg-Cu Alloys in a Wide Range of Quenching Rates Using Small angle X-ray Scattering
}

\section{Yong Zhang ${ }^{1,2 *}$, Daniele Pelliccia ${ }^{3,4}$, Benjamin Milkereit ${ }^{5,6}$, Nigel Kirby ${ }^{4}$, Marco J. Starink ${ }^{7}$, Paul A. Rometsch ${ }^{2}$}

${ }^{1}$ School of Materials Science and Engineering, Central South University, Changsha Hunan, China

2 Department of Materials Science and Engineering, Monash University, Clayton, Victoria, Melbourne, Australia

${ }^{3}$ School of Science RMIT University, Melbourne, Victoria, Australia

${ }^{4}$ Australian Synchrotron, Clayton, Victoria, Australia

${ }^{5}$ Chair of Materials Science, Faculty of Marine Technology and Mechanical Engineering, University of Rostock, 18051 Rostock, Germany

$6{ }^{\circ}$ CALOR - Research Competence Centre Calorimetry and Thermal Analysis Rostock, Faculty for Interdisciplinary Research, Department Light, Life and Matter, University of Rostock, 18051 Rostock, Germany

${ }^{7}$ Materials Research Group, Faculty of Engineering and the Environment, University of Southampton, SO17 1BJ Southampton, United Kingdom
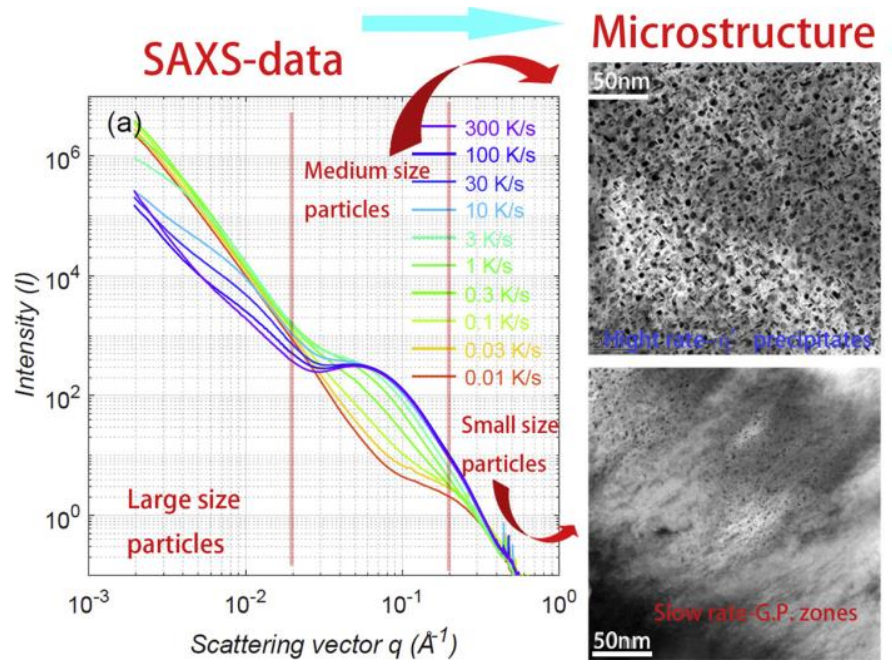

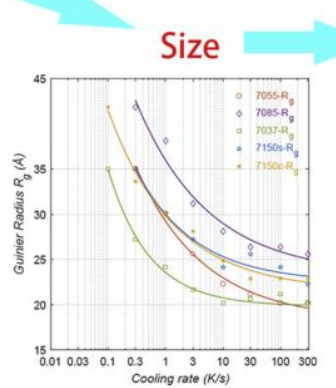
Volume fraction

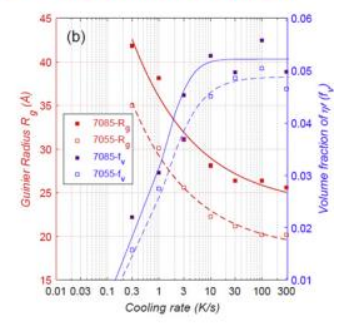




\begin{abstract}
The volume fraction of age hardening precipitates upon peak ageing after different quenching rates has been studied for five $\mathrm{Al}-\mathrm{Zn}-\mathrm{Mg}-\mathrm{Cu}$ alloys using small angle $\mathrm{X}$-ray scattering (SAXS) and transmission electron microscopy (TEM). The rate of change in volume fraction of the $\eta^{\prime}$ age hardening precipitates as a function of cooling rate was used to evaluate changes in the degree of solute supersaturation, and hence the quench sensitivity of the Al-Zn$\mathrm{Mg}-\mathrm{Cu}$ alloys. The results demonstrate that the amount of $\eta^{\prime}$ age hardening precipitates can be changed by varying the thermomechanical processing history or the alloy compositions. It was found that the volume fraction of age hardening $\eta^{\prime}$ precipitates in $\mathrm{Al}-\mathrm{Zn}-\mathrm{Mg}-\mathrm{Cu}$ alloys increases with increasing cooling rates and increasing Mg content as well as that the size of $\eta^{\prime}$ precipitates is related to the $\mathrm{Cu} / \mathrm{Mg}$ ratio.
\end{abstract}

Keywords: Quench sensitivity; small angle X-ray scattering; age hardening precipitates; volume fraction; $\mathrm{Al}-\mathrm{Zn}-\mathrm{Mg}-\mathrm{Cu}$ alloys

\title{
1. Introduction
}

The $\mathrm{Al}-\mathrm{Zn}-\mathrm{Mg}(-\mathrm{Cu})$ series aluminium alloys demonstrate the strongest age hardening capability among all aluminium alloys [1]. These alloys, as well as other age hardening alloys, such as Al-Cu and Al-Mg-Si alloys, may suffer from quench sensitivity problems during the solution-quenching process [2]. Previous studies have shown that the quench sensitivity problem is due to the formation of quench-induced precipitates during "slow" cooling, which deplete solute from the matrix and thereby decrease the subsequent age hardening potential [3, 4]. Consequently, the alloy cannot achieve desirable properties such as fracture toughness, stress corrosion resistance and mechanical strength [5]. This quench sensitivity phenomenon 
is especially critical for thick gauge products where the centre layers always experiences a slower cooling rate compared to the surface layers $[6,7]$.

Recently, Milkereit et al. [8] and Zhang et al. [7] have developed continuous cooling precipitation (CCP) diagrams for $\mathrm{Al}-\mathrm{Mg}-\mathrm{Si}$ and $\mathrm{Al}-\mathrm{Zn}-\mathrm{Mg}(-\mathrm{Cu})$ alloys, respectively. These $\mathrm{CCP}$ diagrams show the different quench-induced reaction regions, thereby enabling selection of cooling paths that avoid unwanted quench-induced reactions and limit distortion and residual stresses at the same time.

Based on a recent body of work published by the same group of authors [7, 9-11], it is known, that the different quench induced phase particles (which precipitate at different temperatures and substantially different time scales), have a wide range of dimensions. For instance, at low cooling rates $\mathrm{S}$-phase $\left(\mathrm{Al}_{2} \mathrm{CuMg}\right)$ particles, precipitating at temperatures of about 450 to $350{ }^{\circ} \mathrm{C}$, have dimensions of up to some tens of $\mu \mathrm{m}$. The $\eta$-phase particles (based on the $\operatorname{Mg}(\mathrm{Zn}, \mathrm{Al}, \mathrm{Cu})_{2}$ composition), which precipitate during cooling at temperatures of about 350 to $250{ }^{\circ} \mathrm{C}$, have diameters of typically several $100-500 \mathrm{~nm}$. The $\mathrm{Y}$-phase platelets (enriched in $\mathrm{Cu}$ and $\mathrm{Zn}$ ), precipitating at faster rates and temperatures of about 250 to $150{ }^{\circ} \mathrm{C}$, have thicknesses which might be as thin as just a few atomic layers [12]. However, their lateral dimension still reaches up to the $\mu \mathrm{m}$ range. At even lower temperatures and cooling rates slightly below the critical cooling rate, cluster formation is likely to occur during cooling [13]. The CCP diagrams have shown that the critical cooling rate (CCR) for a highly concentrated $\mathrm{Al}-\mathrm{Zn}-\mathrm{Mg}-\mathrm{Cu}$ alloy can be in the order of several hundred $\mathrm{K} / \mathrm{s}[7,9,10]$. For a conventional industrial application, it is very difficult to achieve such fast cooling rate conditions, particularly for thicker products, and it is therefore of interest to understand quantitatively how much solute was lost over a specific cooling path since this determines how much solute is 
still available for subsequent ageing. By understanding the quantitative relationship between the remaining solute and the cooling rate, one can successfully predict mechanical properties after quenching.

Thus, the analysis of quench induced precipitation in a dynamic cooling rates range of several orders of magnitude requires detection of particles ranging from some tens of $\mu \mathrm{m}$ down to few $\mathrm{nm}$ or even less. It therefore is difficult to quantitatively analyse the volume fractions of these phases directly, as their volume fractions and sizes change significantly with cooling rate. Starink and co-workers have recently developed a modeling approach to predict the volume fractions of precipitates from diffusion-controlled reactions during quenching of several Al$\mathrm{Mg}-\mathrm{Si}$ and $\mathrm{Al}-\mathrm{Zn}-\mathrm{Mg}-\mathrm{Cu}$ alloys $[10,14]$. However, experimental verification of the model output was limited to coarse particles visible using optical and scanning electron microscopes.

The small angle X-ray scattering (SAXS) technique offers a non-destructive bulk method to quantitatively analyse the shape, size and volume fraction of nano-scale age hardening precipitates [15-19]. However, the SAXS technique is limited to the detection of small (namometer sized) particles in the order of $100 \mathrm{~nm}$, depending on the exact setup of the experiment. Thus, SAXS can provide information on volume fraction and size distribution of age hardening $\eta$ ' precipitates which is directly related to achievable strength.

In this work the SAXS is used on $\mathrm{Al}-\mathrm{Zn}-\mathrm{Mg}-\mathrm{Cu}$ alloys which are solutionised and quenched in a wide range (covering nearly five orders of magnitude) and finally artificially aged in a same regime. The artificial ageing stabilizes samples allowing SAXS analysis at extended times. Applying this indirect way, SAXS allows to draw conclusions on how variations of the 
cooling rate in a wide dynamic range could affect the properties of studied alloys. The SAXS experiments are supported by transmission electron microscopy analysis.

\section{Materials and methods}

The compositions of the studied alloys are given in Table 1 (in at\%). Alloy $7150 \mathrm{C}$ represents the centre layer of a commercially processed $80 \mathrm{~mm}$ thick AA7150 plate, while alloy $7150 \mathrm{~S}$ represents the surface layer of the same commercially processed AA7150 plate. It is shown that the chemical compositions of the surface layer and the centre are slightly different. The alloys 7055,7085 and 7037 are lab-produced alloys with systematically changing $\mathrm{Zn} / \mathrm{Mg}$ ratio or $\mathrm{Cu}$ content, and are named here according to the nominal or nearest AA standard for common $\mathrm{Al}-\mathrm{Zn}-\mathrm{Mg}-\mathrm{Cu}$ alloys.

Table 1: Actual measured chemical compositions of the studied Al-Zn-Mg-Cu alloys (at\%)

\begin{tabular}{c|ccccccccc}
\hline Alloy & $\mathrm{Fe}$ & $\mathrm{Si}$ & $\mathrm{Zn}$ & $\mathrm{Mg}$ & $\mathrm{Cu}$ & $\mathrm{Zr}$ & $\mathrm{Zn} / \mathrm{Mg}$ & $\mathrm{Cu} / \mathrm{Mg}$ & $\mathrm{Zn}+\mathrm{Mg}+\mathrm{Cu}$ \\
\hline $7150 \mathrm{C}$ & 0.03 & 0.01 & 2.79 & 2.54 & 0.96 & 0.03 & 1.10 & 0.38 & 6.29 \\
$7150 \mathrm{~S}$ & 0.03 & 0.01 & 2.65 & 2.40 & 0.91 & 0.03 & 1.10 & 0.38 & 5.96 \\
7055 & 0.02 & 0.03 & 3.56 & 2.36 & 0.90 & 0.04 & 1.51 & 0.38 & 6.82 \\
7085 & 0.02 & 0.07 & 3.58 & 1.72 & 0.93 & 0.04 & 2.08 & 0.54 & 6.23 \\
7037 & 0.01 & 0.12 & 3.40 & 1.60 & 0.41 & 0.04 & 2.13 & 0.26 & 5.41 \\
\hline
\end{tabular}

All cast alloys were placed into an air circulation furnace for a homogenisation treatment of 48 hours at $460{ }^{\circ} \mathrm{C}$ to avoid possible incipient melting during subsequent solution treatment. A two-step solution treatment was carried out subsequently, a first soaking step at $460{ }^{\circ} \mathrm{C}$ for 1 hour followed by another soaking at $480{ }^{\circ} \mathrm{C}$ for an additional hour. Linear cooling was carried out using different differential scanning calorimeter (DSC) devices or a quenching 
dilatometer covering a wide range of cooling rates. Solution treatment and cooling was done in Rostock, Germany. The detailed cooling methods and recorded CCP diagrams for all studied alloys can be found within references [7, 9-11]. The specimens were then aged at $120{ }^{\circ} \mathrm{C}$ for 24 hours immediately after quenching. This allowed stabilization of the microstructural state for transportation to the Australian Synchrotron and sample preparation.

SAXS samples were prepared as $3 \mathrm{~mm}$ discs punched directly from the aged DSC/dilatometer samples. The measurements were carried out on the SAXS/WAXS beamline at the Australian Synchrotron. X-rays generated by an undulator are focused and monochromated by a $\mathrm{Si}$ (111) double crystal monochromator. Two values of X-ray energy were used for the $\mathrm{Cu}$ edge (8400 $\mathrm{eV}$ and $8974 \mathrm{eV}$ ) and two values for the $\mathrm{Zn}$ edge $(9200 \mathrm{eV}$ and $9655 \mathrm{eV})$. Because of different phases emerging at different cooling rates, a large range of precipitate sizes ranging from several $\mathrm{nm}$ up to the micrometer scale was expected. Therefore the experiment included data acquisition with two sample-camera distances. We used both short $(951.1 \mathrm{~mm})$ and long (7241.3 $\mathrm{mm}$ ) distance from the same samples, which are sensitive to different regions in the $q$-space, and hence different precipitates size. Data were collected with a two-dimensional (2D) pixel array detector (Pilatus1M Dectris, with pixel size $\mathrm{p}=172 \mu \mathrm{m}$ ) and were then merged to yield large $q$-range information.

All SAXS samples had a thickness of $200 \pm 5 \mu \mathrm{m}$ and were placed in a custom-designed holder. The X-ray beam was programmed for consecutive measurements before energy was changed to the next value. Each sample was measured four times at different locations at a given energy level for the purpose of minimising statistical error. 
Samples for TEM were prepared directly from the SAXS samples by grinding them to a thickness of $\sim 120 \mu \mathrm{m}$ for twin-jet electro-polishing using a Tenupol-5 with a solution of $33 \%$ nitric acid and $67 \%$ methanol, at a temperature of $-30{ }^{\circ} \mathrm{C}$. Since samples are quenched under different cooling conditions, causing the microstructures of the samples to differ substantially, the samples studied in this work have different electrical potentials. For optimal TEM sample preparation by electropolishing, it thus proved necessary to use different voltages in the process and electropolishing voltages in the range $8-13.8 \mathrm{~V}$ were used. The TEM observations were undertaken using an FEI Tecnai G2 T20 TEM at $200 \mathrm{kV}$, equipped with a Bruker Quantax 200 TEM X-ray spectrometer.

\section{Results and discussion}

\subsection{Scattering curves and microstructures}

Since five $\mathrm{Al}-\mathrm{Zn}-\mathrm{Mg}-\mathrm{Cu}$ alloys with different chemical compositions were investigated in the cooled and artificially aged condition at four different energy levels, two different camera lengths and 10 different cooling rate conditions ranging from $0.01 \mathrm{~K} / \mathrm{s}$ to $300 \mathrm{~K} / \mathrm{s}$, a large amount of data processing was involved. The current paper uses SAXS data mainly from alloy 7055 to demonstrate the data evaluation. This alloy has the highest alloying concentration and is thus expected to have the most significant quench sensitivity over the studied cooling rate range.

The scattered intensity from the samples was first calibrated against the scattering of a reference glassy carbon (GC) plate. All scattering patterns were background corrected and normalised to the sample thickness. 
After the thickness correction, the intensity was corrected by the Laue background [19], which is the background intensity due to scattering from a disordered solid solution (not from the precipitates). This differs from sample to sample as the solid solution changes and it must therefore be subtracted from the scattered signal [19].

The Laue intensity follows the relation:

$$
I_{\text {raw }} \boldsymbol{q}^{\mathbf{4}} \equiv\left(K_{p}+I_{L a u e} q^{\mathbf{4}}\right)_{q \rightarrow \infty}
$$

Equation 1

Where $I_{\text {raw }}$ is the raw data after thickness normalistion, $K_{\mathrm{p}}$ is the Porod constant and $q$ is the scattering vector. As a result, $I_{\text {Laue }}$ can be determined by plotting $I_{\text {raw }} q^{4}$ vs. $q^{4}$ (see Fig. 1(a)). The slope of the linear part in Fig. 1(a) is equal to $I_{\text {Laue }}$ and can be subtracted from $I_{\text {raw }}$ to obtain scattering intensity due to the precipitates. Fig. 1(b) shows $I_{\text {Laue }}$ the as a function of cooling rates for all studied alloys, and it suggest the $I_{\text {Laue }}$ generally increases with increasing cooling rate. However, this would need further confirmation work, particularly it might depend on the size of objects.

Fig. 2(a) shows a plot of the intensity versus the scattering vector plotted after the Laue correction. The Guinier radius of the particles is estimated as the reciprocal to the scattering vector according to Guinier approximation [19]. Previous studies have shown that the Guinier radius is very close to the mean radius of the age hardening precipitates in the $\mathrm{Al}-\mathrm{Zn}-\mathrm{Mg}$ system [20]. The Laue corrected intensity (Fig. 2(a)) curves can then be approximated by three broad regions in terms of precipitate size:

1. Small $q$ for large precipitate sizes $\left(q \leq \sim 0.02 \AA^{-1}\right)$.

2. Medium $q$ for medium precipitate sizes $\left(\sim 0.02<q \leq \sim 0.2 \AA^{-1}\right)$. 
3. Large $q$ for small precipitate sizes $\left(q>\sim 0.2 \AA^{-1}\right)$.

The three regions suggest three different families of precipitates, mainly distinguished by their size. The large particles with small scattering vector $q$ may correspond to quench-induced particles (e.g. $\mathrm{Al}_{2} \mathrm{CuMg}, \mathrm{Mg}(\mathrm{Zn}, \mathrm{Al}, \mathrm{Cu})_{2}$ ), dispersoids (e.g. $\mathrm{Al}_{3} \mathrm{Zr}$ ) and/or intermetallic particles (e.g. $\mathrm{Al}_{7} \mathrm{Cu}_{2} \mathrm{Fe}, \mathrm{Mg}_{2} \mathrm{Si}$ ). Since their sizes range from several hundred $\mathrm{nm}$ to several $\mu \mathrm{m}$, some of the scattering signals from these particles are beyond the detection capability of the SAXS instrument. The medium sized particles with medium $q$ most likely correspond to the $\eta^{\prime}$ age hardening precipitates $[21,22]$. Since these particles precipitate homogenously during the ageing process, they have a relatively uniformly distributed size and shape. Therefore, the scattering signal from these particles can be quantitatively studied within the detection range. A "structure factor" effect was observed for the medium size particles ( $q \sim$ 0.02-0.06 $\left.\AA^{-1}\right)$. It is the interference effect between the X-rays scattered from individual particles that can be measured when the density of the particles is very high. When this effect becomes more significant, it indicates that a higher number density of medium size particles precipitated in the fast cooling rate range. Small size particles with large scattering vector $q$ may correspond to G.P. zones or solute clusters. A recent paper has pointed out that GP zones can form during nonlinear cooling [13]. Interestingly, they can be clearly detected during some relatively slow cooling rate conditions $(0.1,0.03$ and $0.01 \mathrm{~K} / \mathrm{s}$ for alloy 7055$)$ in the current work. However, because of their extremely small size, the background becomes very noisy in the very large $q$ range.

Fig. 2 (b) reveals that, within the $q$ range of $\sim 0.05$ to $0.25 \AA^{-1}$, the curves follow the $q^{\alpha}$ relationship, and the $\alpha$ values change gradually from -4.0 to -2.8 with increasing cooling rate in this $q$ range. A similar trend was detected for all the other studied alloys, as indicated in Fig. 
2 (c). At cooling rates of $\leq 0.1 \mathrm{~K} / \mathrm{s}$, the curves appear different, indicating that the detected objects may be changing in a different manner.

Figure 3 shows that the morphology of the $\eta$ ' precipitates formed after ageing changes with different solution-quenching process. The TEM images show that the $\eta$ ' precipitates are small and rounded at the faster cooling rates and larger and more elongated at the slower cooling rates. It seems that the morphology of the $\eta^{\prime}$ precipitates is associated with $\alpha$ values. The selected area diffraction (SAD) patterns in Fig. 3 (b) confirms that even though the morphology changed, all precipitates are still $\eta$ ' phase. The number density of $\eta$ ' precipitates is significantly higher at faster cooling rates than at slower cooling rates. It is also shown in Fig. 3(e) that no $\eta$ ' precipitates can be observed at the very slow cooling rate of $0.03 \mathrm{~K} / \mathrm{s}$. The corresponding SAD pattern at lower right corner indicates that the particles are G.P.I zones $[23,24]$. These results are also consistent with the SAXS results in Fig.2 (a), where it is evident that at cooling rates of $\leq 0.1 \mathrm{~K} / \mathrm{s}$ scattering data exhibit different curve shapes than for other cooling conditions. Fig. 3(e) shows that a high number density of G.P.I zones can be observed for these slow cooling rates after ageing. They are generally considered to form during natural ageing - in this case possibly during the extended natural ageing that the SAXS and TEM samples were exposed to room temperature after artificial ageing. However, Schloth et al. showed that G.P.I zones can also form during the cooling process [13]. Liu et al. also demonstrated that a large amount of stable G.P.I zones can be found prior to artificial ageing in slowly cooled samples [25]. In the current experiments, the distribution of G.P.I zones did not significantly change with cooling rates. This could be due to low vacancy concentration and low solute concentration within the very slow quenched samples. The quenched-in vacancies annihilate sharply with decreasing cooling rates. According to Falahati et al. the 
vacancy density of aluminium alloys can change from $5 \times 10^{-5}$ for fast quench to $2 \times 10^{-9}$ for very slow quench rate $[26,27]$. The low solute concentrations, which are caused by the quench induced phases consuming the solutes [7-9, 12], cause a reduced driving force for continued age hardening precipitates formation during artificial ageing.

It should be noted that whilst the present analysis of precipitate shape using SAXS data shows trends that are consistent with literature data, the present method of interpretation of SAXS data needs further research. Particularly the present simple linear fitting needs to be critically assessed as it may be an oversimplification.

\subsection{Kratky plot}

In order to calculate the volume fraction of $\eta^{\prime}$ precipitates that form during ageing after exposure to different cooling rate conditions, Kratky plots $\left(I . q^{2} v s . q\right)$ were produced as shown in Fig. 4. In the Kratky plots, a specific peak corresponds to a given family of particles within the studied samples. In the low $q$ region shown in Fig. 4(a), there is a group of broad peaks in the cooling rate range from 1 to $100 \mathrm{~K} / \mathrm{s}$, and the area under the peak decreases with increasing cooling rate. As discussed before, this group of particles corresponds mainly to the combination of intermetallic phases and quench-induced phases, though only the quenchinduced phases change with cooling rate. The position of $q_{\max }$ can be used to determine the Guinier Radius $R_{g}=\sqrt{3} / q_{m}$ [22]. Fig. 4(a) shows that the position of the maximum $q$ value $\left(q_{\max }\right)$ is shifting from very small $\left(\sim 0.002 \AA^{-1}\right)$ to much larger but still relatively small $q$ values $\left(\sim 0.01 \AA^{-1}\right)$, which indicates that both the volume fraction (area under the peak) and the average size (position of $q_{\max }$ ) of these quench-induced precipitates decrease with increasing cooling rate. They are quench-induced precipitates with $R_{\mathrm{g}}>170 \AA$. In the relatively slow 
cooling rate range $(<1 \mathrm{~K} / \mathrm{s})$, their volume fraction and size are too large to be detected by this SAXS technique.

Fig. 4(b) shows the Kratky plot for the full range of scattering vector $q$. The main peak here represents another group of particles, whose $q_{\max }$ is shifting from high to low $q$ values with decreasing cooling rate, indicating that the average size of these particles increases with decreasing cooling rate. The TEM results in Fig. 3 have shown that they are age hardening $\eta^{\prime}$ precipitates, and it is evident from both the SAXS and TEM results that the volume fraction of these precipitates tends to decrease with decreasing cooling rate.

In the high $q$ region, expanded in Fig. 4(c), it is evident that there is another peak at the cooling rate of $<0.1 \mathrm{~K} / \mathrm{s}$ with $q_{\max }$ at $\sim 0.23 \AA^{-1}$. This corresponds to $R_{\mathrm{g}} \sim 7.5 \AA^{-1}$ and appears to represent the G.P.I zones that were detected by SAD in the TEM at the cooling rate of 0.03 $\mathrm{K} / \mathrm{s}$ (Fig. 3). It is interesting to note that this peak is most pronounced at the cooling rate of 0.1 $\mathrm{K} / \mathrm{s}$. Its intensity decreases with decreasing cooling rate, suggesting that natural ageing occurs during room temperature storage (in this case $>2$ months) after $24 \mathrm{~h}$ of artificial ageing at $120^{\circ} \mathrm{C}$, and that the amount of the zones is also associated with the solute concentration after the solution-quenching process. It is possible that there is also a G.P.I zones peak at cooling rate of $>0.1 \mathrm{~K} / \mathrm{s}$, but that this is not easy to see due to overlap with the $\eta^{\prime}$ peak. It appears in the TEM results in Fig.3, that G.P.I zones may also be present between the $\eta$ ' precipitates.

The Guinier radius of these $\eta$ ' precipitates are plotted against the cooling rate for all the studied alloys in Fig. 5. It is interesting to note that the radius of the $\eta$ ' precipitates depend on alloy composition. In alloy 7037 , with the smallest $\mathrm{Cu} / \mathrm{Mg}$ ratio of 0.26 amongst the alloys studied, the smallest $\eta$ ' precipitates formed during ageing. Alloy 7085, corresponding to the largest 
$\mathrm{Cu} / \mathrm{Mg}$ ratio of 0.54 , has the largest $\eta$ ' precipitates over the whole range of investigated cooling rates. The sizes of $\eta$ ' precipitates for the other studied alloys are in between these two extremes (corresponding to an intermediate $\mathrm{Cu} / \mathrm{Mg}$ ratio of $\approx 0.38$ ). The only exception is that alloy 7055 has very small $\eta$ ' precipitates in the relatively fast cooling rate range (comparable to alloy 7037 at $\geq 30 \mathrm{~K} / \mathrm{s}$ ), although their size increases significantly with decreasing cooling rate. The underlying mechanism of how the $\mathrm{Cu} / \mathrm{Mg}$ ratio could affect the size of the $\eta$ ' precipitates is still unclear at this moment and is worthy of future investigation as this appears to have not been reported previously.

The volume fraction of the scattering precipitates is proportional to the invariant or integrated intensity $Q$, which is proportional to the area under the scattering curve in the Kratky plot. Assuming a two-phase model, separated by a sharp interface, the volume fraction can be calculated as [28]:

$$
Q_{0}=\int_{0}^{\infty} I q^{2} d q=2 \pi^{2}(\Delta \rho)^{2} f_{v}\left(1-f_{v}\right)
$$

\section{Equation 2}

Measuring the whole scattering curve from 0 to infinity is not possible due to a limited available $q$-range in the experimental measurements. Therefore, extrapolations for the low $q$ and high $q$ regions are necessary $[19,20]$.

In the low $q$ region, a linear extrapolation from the first measured point $q_{0}$ to 0 can be performed. This is only valid if there is no strong parasitic scattering, e.g. double Bragg scattering or scattering from large particles. Since no $\eta$ ' precipitates can be detected at cooling rates of $\leqslant 0.1 \mathrm{~K} / \mathrm{s}$, the $Q_{0}$ calculation is only valid for cooling rates of $>0.1 \mathrm{~K} / \mathrm{s}$ for alloy 7055 . In the high $q$ region, the integral can be extended to infinity by fitting the scattered intensity with Porod's law, giving [19, 21, 22]: 


$$
Q_{0} \approx \frac{I\left(q_{1}\right) q_{1}^{3}}{2}+\int_{q_{1}}^{q_{2}} I(q) q^{2} d q+\frac{K_{p}}{q_{\max }} \quad \text { Equation } 3
$$

According to Equation 2, for a given value of integrated intensity, a relationship between composition $(\Delta \rho)$ and volume fraction $\left(f_{v}\right)$ can be drawn. $\Delta \rho$ is the electron density contrast between the precipitates and the matrix $\left|\Delta \rho_{p}-\Delta \rho_{m}\right|$. In order to calculate the volume fraction $f_{v}, \Delta \rho$ and $Q_{0}$ are required but only $Q_{0}$ is known so far. As a result, the composition of the precipitates needs to be evaluated using the Anomalous Small Angle X-ray Scattering (ASAXS) technique $[19,21,29]$. It should be noted that in ASAXS there is a strong lever effect, which amplifies inaccuracies on the absolute scaling of the intensity when the scattering energy is too close to the edges. Thus if ASAXS is applied more energy levels should be chosen to eliminate such uncertainties in future work.

According to equations 2 , it is necessary to obtain compositional information for both the precipitates $\left(C_{i}^{P}\right)$ and matrix $\left(C_{i}^{m}\right)$. Based on previous literature and atom probe tomography (APT) results $[21,22]$, we hereby assume $\mathrm{Mg}(\mathrm{Zn}, \mathrm{Al}, \mathrm{Cu})_{2}$ stoichiometry for the $\eta^{\prime}$ phase and that $C_{M g}^{p}=33 \%$ and $C_{A l}^{p}=15 \%$ for all cooling conditions.

The $\mathrm{Zn}$ and $\mathrm{Cu}$ concentrations in the matrix are generally small compared to those in the precipitates. Although there are some data on the matrix concentrations in $\mathrm{Al}-\mathrm{Zn}-\mathrm{Mg}$ alloys in different ageing conditions, the samples in those studies were generally water quenched i.e. quenched at a high rate $[21,22]$, and hence substantially different from our samples which were aged after different cooling rates. The present work shows that the Laue background, which is due to a disordered solid solution i.e. the Al-rich matrix phase [19] increases clearly with increasing cooling rate (as shown in Fig. 1(a)), which is an indication that the matrix composition in the cooled and artificially aged condition increases with increasing cooling 
rates. We therefore calculated the matrix concentration according to the Laue background intensity for different cooling conditions, as shown in Fig. 6:

$$
C_{i j}^{m}=\frac{I_{\text {Laue }}^{i j}}{I_{\text {Laue }}^{\text {max }}} * C_{i}^{\text {max }}
$$

\section{Equation 4}

where $j$ is cooling rate of element $i(\mathrm{Zn}$ and $\mathrm{Cu}$ in this case $)$, and $\mathrm{C}_{\mathrm{i}}^{\max }$ is the maximum matrix concentration that can be achieved in the cooled and artificially aged condition for a given alloy, which is equal to actual measured chemical compositions.

It is demonstrated that the matrix concentration decreases slightly with decreasing cooling rate. As a result, the larger $\eta^{\prime}$ precipitates in the slow cooled conditions in Fig. 3 are most likely due to the lower matrix concentration remaining after slow cooling both before and after ageing. This phenomenon is frequently observed adjacent to precipitate free zone (PFZ) regions, where a lower matrix concentration leads to relatively large $\eta$ ' precipitates [30-32].

\subsection{Volume fraction of age hardening precipitates}

The volume fraction of age hardening $\eta$ ' precipitates can be calculated by incorporating the compositions of the precipitates and matrix. Figure 7 shows the calculated volume fractions together with the Guinier radii for the different alloys. In order to understand the different quench sensitivity behaviours, the data have been divided into three groups.

It is shown in Fig. 7 (a) that the first group includes alloys $7150 \mathrm{C}$ and $7150 \mathrm{~S}$, which represent the centre layer and surface layers of the same commercially processed AA7150 plate, respectively. They have experienced a different thermomechanical processing history, as the surface is always exposed to more severe deformation than the centre layer. Previous studies have shown that the surface layer and centre layer exhibit different quench sensitivity 
behaviours [6], which was mainly attributed to different recrystallisation fractions after solution treatment. The quench-induced phases precipitate preferentially on dispersoids in recrystallised grains. Therefore, the higher degree of recrystallisation near the plate surface causes a higher quench sensitivity. The results here in Fig. 7 (a) confirm that alloy $7150 \mathrm{~S}$ is more quench sensitive at cooling rates of $<30 \mathrm{~K} / \mathrm{s}$, as the volume fraction of $\eta$ ' precipitates decreases more rapidly with decreasing cooling rate for $7150 \mathrm{~S}$ than for $7150 \mathrm{C}$. At cooling rates of $>30 \mathrm{~K} / \mathrm{s}$, the volume fraction of $\eta$ ' precipitates decreases slightly with increasing cooling rate. This could be due to some Y-phase precipitation during cooling, which would slightly decrease the amount of solute that remains for $\eta$ ' precipitates during subsequent ageing [12].

The second group including alloys 7055 and 7085, is shown in Fig. 7 (b). Table 1 shows that these two alloys have similar alloy compositions except that alloy 7085 has a lower $\mathrm{Mg}$ content. It is shown that alloy 7085 contains a higher volume fraction of age hardening $\eta$ ' precipitates over the entire cooling rate range from $0.3 \mathrm{~K} / \mathrm{s}$ to $300 \mathrm{~K} / \mathrm{s}$. In other words, there is more solute available in the right stoichiometric ratio for $\eta$ ' precipitate formation during ageing after the solution-quenching process for alloy 7085 as compared to alloy 7055 . Furthermore, the volume fraction of $\eta$ ' precipitates starts to drop from $<30 \mathrm{~K} / \mathrm{s}$ for alloy 7055 but only from $<10$ $\mathrm{K} / \mathrm{s}$ for alloy 7085 , and alloy 7085 still has the same volume fraction of $\eta^{\prime}$ precipitates for a cooling rate of $3 \mathrm{~K} / \mathrm{s}$ as alloy 7055 has for a cooling rate of $10 \mathrm{~K} / \mathrm{s}$. It is therefore evident that alloy 7085 has a lower quench sensitivity than alloy 7055 .

For all the cooling rates that were studied, the Guinier radius of the $\eta^{\prime}$ precipitates is larger for alloy 7085 than for alloy 7055 . This was already discussed in the context of Fig. 5. Together with the composition information, it is concluded that the quench sensitivity increases with 
increasing $\mathrm{Mg}$ content and decreasing $\mathrm{Zn} / \mathrm{Mg}$ ratio. The result is consistent with some other publications $[33,34]$.

The third group includes alloys 7085 and 7037, and the variation in size/volume fraction of age hardening $\eta^{\prime}$ precipitates as a function of cooling rate is shown in Fig. 7 (c). It is demonstrated that the Guinier Radius of the $\eta$ ' precipitates for alloy 7085 is significantly larger than that for alloy 7037 for all cooling rates. The volume fraction of $\eta$ ' precipitates for alloy 7085 is significantly larger than that of alloy 7037 in the cooling rate range of $\geqq 3 \mathrm{~K} / \mathrm{s}$. This is mainly due to the fact that alloy 7085 contains more $\mathrm{Zn}, \mathrm{Mg}$ and $\mathrm{Cu}$, i.e. alloy 7085 has a higher total alloying content than alloy 7037 . Fig. 7 (c) demonstrates that alloy 7037 experiences a relatively small change in volume fraction of $\eta^{\prime}$ precipitates over the cooling rate range of $1-100 \mathrm{~K} / \mathrm{s}$, while over the same cooling rate range, the volume fraction of $\eta^{\prime}$ precipitates for alloy 7085 decreases dramatically from $5 \%$ to $<3 \%$. At cooling rates of $\leqq 10$ $\mathrm{K} / \mathrm{s}$, the volume fraction of $\eta^{\prime}$ precipitates for alloy 7085 drops so dramatically that it drops below that in alloy 7037 at cooling rates below about $1 \mathrm{~K} / \mathrm{s}$. It is therefore concluded that alloy 7037 is less quench sensitive than alloy 7085 . However, the maximum attainable strength of 7037 is lower than that of alloy 7085 because of the lower amount of age hardening $\eta^{\prime}$ precipitates (for cooling rates of $\geqq 1 \mathrm{~K} / \mathrm{s}$ ).

\section{Conclusions}

SAXS was used to analyse the volume fractions of age hardening ๆ' precipitates for four Al$\mathrm{Zn}-\mathrm{Mg}-\mathrm{Cu}-\mathrm{Zr}$ alloys subjected to different continuous cooling conditions, allowing, for the first time, direct analysis of the volume fractions and Guinier radius of nanometer sized particles through a range of alloys quenched at a wide range of cooling rates. 
For a thick 7150 plate experiencing more deformation at the plate surface than centre, the quench sensitivity is higher at the plate surface. For similarly concentrated alloys, the quench sensitivity of the $\mathrm{Al}-\mathrm{Zn}-\mathrm{Mg}-\mathrm{Cu}$ alloys increases with increasing $\mathrm{Mg}$ content when this decreases the $\mathrm{Zn} / \mathrm{Mg}$ ratio below about 2. For alloys with different total alloying contents, the quench sensitivity increases with increasing alloying content as long as the $\mathrm{Zn} / \mathrm{Mg}$ ratio is amenable to $\eta$ ' formation during subsequent ageing.

For alloy 7055, both the Guinier Radius and the aspect ratio of the age hardening $\eta$ ' precipitates increase with decreasing cooling rate from 100 to $0.1 \mathrm{~K} / \mathrm{s}$. At cooling rates of below $0.1 \mathrm{~K} / \mathrm{s}$, no $\eta^{\prime}$ precipitates were observed in the peak aged condition. Instead, only G.P. I zones were observed. Alloys with higher $\mathrm{Cu} / \mathrm{Mg}$ ratios have larger sizes of $\eta$ ' precipitates.

Apart from the quench sensitivity evaluation, the SAXS results also demonstrated how the Laue background and matrix composition varies for different cooling conditions.

\section{Acknowledgements}

The authors thank the Aluminium Corporation of China Ltd. (Chalco) for providing materials. The continuous cooling experiments were performed at the Polymer Physics Group at the University of Rostock, and Prof. Olaf Kessler and Prof. Christoph Schick are gratefully acknowledged for helpful discussions. Prof. Alexis Deschamps and Prof. Chris Hutchinson are also greatly thanked for fruitful discussions. All the microstructural characterisation work was performed on instruments within the Monash Centre for Electron Microscopy. SAXS data was acquired on the SAXS/WAXS beamline at the Australian Synchrotron, Victoria, Australia. 


\section{References:}

[1] I.J. Polmear, Light Alloys: Metallurgy of the Light Metals, 4th ed., Melbourne, 2006.

[2] J.E. Hatch, Aluminum: Properties and Physical Metallurgy Aluminum Association Inc. and ASM International1984.

[3] S. Liu, X. Zhang, M. Chen, J. You, X. Zhang, Effect of Zr Content on Quench Sensitivity of AlZnMgCu alloys, Trans. Nonferrous Met. Soc. China 17 (2007) 787-792.

[4] X. Zhang, W. Liu, S. Liu, M. Zhou, Effect of Processing Parameters on Quench Sensitivity of an AA7050 sheet, Mater. Sci. Eng., A 528 (2011) 795-802.

[5] D. Dumont, A. Deschamps, Y. Bréchet, A Model for Predicting Fracture Mode and Toughness in 7000 Series Aluminum alloys, Acta Mater. 52 (2004) 2529-2540.

[6] Y. Zhang, C. Bettles, P.A. Rometsch, Effect of recrystallisation on $\mathrm{Al}_{3} \mathrm{Zr}$ dispersoid behaviour in thick plates of aluminium alloy AA7150, J. Mater. Sci. 49 (2014) 1709-1715.

[7] Y. Zhang, B. Milkereit, O. Kessler, C. Schick, P.A. Rometsch, Development of continuous cooling precipitation diagrams for aluminium alloys AA7150 and AA7020, J. Alloys Compd. 584 (2014) 581-589.

[8] B. Milkereit, N. Wanderka, C. Schick, O. Kessler, Continuous cooling precipitation diagrams of Al-Mg-Si alloys, Mater. Sci. Eng., A 550 (2012) 87-96.

[9] B. Yang, B. Milkereit, Y. Zhang, P.A. Rometsch, O. Kessler, C. Schick, Continuous cooling precipitation diagram of aluminium alloy AA7150 based on a new fast scanning calorimetry and interrupted quenching method, Mater. Charact. 120 (2016) 30-37.

[10] M.J. Starink, B. Milkereit, Y. Zhang, P.A. Rometsch, Predicting the quench sensitivity of $\mathrm{Al}-\mathrm{Zn}-\mathrm{Mg}-\mathrm{Cu}$ alloys: A model for linear cooling and strengthening, Mater. Des. 88 (2015) 958-971.

[11] Y. Zhang, Quench sensitivity of 7xxx series aluminium alloys, Department of materials engineering, Monash University, 2014.

[12] Y. Zhang, M. Weyland, B. Milkereit, M. Reich, P.A. Rometsch, Precipitation of a new platelet phase during the quenching of an Al-Zn-Mg-Cu alloy, Scientific Reports 6(23109) (2016).

[13] P. Schloth, A. Deschamps, C.A. Gandin, J.M. Drezet, Modeling of GP(I) zone formation during quench in an industrial AA7449 75 mm thick plate, Mater. Des. 112 (2016) 46-57.

[14] B. Milkereit, M.J. Starink, Quench sensitivity of Al-Mg-Si alloys: A model for linear cooling and strengthening, Mater. Des. 76 (2015) 117-129.

[15] A. Deschamps, L. Lae, P. Guyot, In situ Small-angle Scattering Study of the Precipitation Kinetics in an Al-Zr-Sc alloy, Acta Mater. 55 (2007) 2775-2783.

[16] A. Deschamps, Y. Bréchet, F. Livet, Influence of copper addition on precipitation kinetics and hardening in Al-Zn-Mg alloy, Mater. Sci. Technol. 15 (1999) 993-1000.

[17] A. Deschamps, Y. Bréchet, Influence of Predeformation on Ageing in an Al-Zn-Mg Alloy-I. Microstructure Evolution and Mechanical Properties, Acta Mater. 47 (1999) 281-292. [18] J.D. Embury, A. Deschamps, Y. Bréchet, The Interaction of Plasticity and Diffusion Controlled Precipitation Reactions, Scr. Mater. 49 (2003) 927-932.

[19] F. De Geuser, A. Deschamps, Precipitate characterisation in metallic systems by smallangle X-ray or neutron scattering, C. R. Phys. 13(3) (2012) 246-256.

[20] M. Dumont, W. Lefebvre, B. Doisneau-Cottignies, A. Deschamps, Characterisation of the composition and volume fraction of $\eta^{\prime}$ and $\eta$ precipitates in an Al-Zn-Mg alloy by a 
combination of atom probe, small-angle X-ray scattering and transmission electron microscopy, Acta Mater. 53 (2005) 2881-2892.

[21] T. Marlaud, A. Deschamps, F. Bley, W. Lefebvre, B. Baroux, Influence of alloy composition and heat treatment on precipitate composition in $\mathrm{Al}-\mathrm{Zn}-\mathrm{Mg}-\mathrm{Cu}$ alloys, Acta Mater. 58(1) (2010) 248-260.

[22] T. Marlaud, A. Deschamps, F. Bley, W. Lefebvre, B. Baroux, Evolution of precipitate microstructures during the retrogression and re-ageing heat treatment of an $\mathrm{Al}-\mathrm{Zn}-\mathrm{Mg}-\mathrm{Cu}$ alloy, Acta Mater. 58(14) (2010) 4814-4826.

[23] G. Sha, A. Cerezo, Early-stage Precipitation in Al-Zn-Mg-Cu alloy (7050), Acta Mater. 52 (2004) 4503-4516.

[24] J.K. Park, A.J. Ardell, Microstructures of the commercial $7075 \mathrm{Al}$ alloy in the T451 and T7 tempers, Metallurgical Transactions A 14(10) (1983) 1957-1965.

[25] S. Liu, C. Li, S. Han, Y. Deng, X. Zhang, Effect of natural aging on quench-induced inhomogeneity of microstructure and hardness in high strength 7055 aluminum alloy, J. Alloys Compd. 625 (2015) 34-43.

[26] P. Lang, A. Falahati, M.R. Ahmadi, P. Warczok, E. Povoden-Karadeniz, E. Kozeschnik, $\mathrm{R}$. Radis, Modeling the influence of cooling rate on the precipitate evolution in $\mathrm{Al}-\mathrm{Mg}-\mathrm{Si}(\mathrm{Cu})$ alloys, Mater. Sci. Technol. 16-20 (2011) 284-291.

[27] A. Falahati, P. Lang, E. Kozeschnik, Precipitation in Al-Alloy 6016 - The Role of Excess Vacancies, Mater. Sci. Forum 706-709 (2011) 317-322.

[28] A. Deschamps, A. Bigot, F. Livet, P. Auger, Y. Brechet, D. Blavette, A comparative study of precipitate composition and volume fraction in an Al-Zn-Mg alloy using tomographic atom probe and small-angle X-ray scattering, Philosophical Magazine A 81(10) (2001) 2391-2414. [29] G. Fribourg, Y. Bréchet, A. Deschamps, A. Simar, Microstructure-based modelling of isotropic and kinematic strain hardening in a precipitation-hardened aluminium alloy, Acta Mater. 59(9) (2011) 3621-3635.

[30] J.K. Park, A.J. Ardell, Microchemical analysis of precipitate free zones in 7075-Al in the T4, T7 and RRA tempers, Acta Metall. Mater. 39(4) (1991) 591-598.

[31] A. Deschamps, G. Texier, S. Ringeval, L. Delfaut-Durut, Influence of cooling rate on the precipitation microstructure in a medium strength Al-Zn-Mg alloy Mater. Sci. Eng., A 501 (2009) 133-139.

[32] J. Tang, H. Chen, X. Zhang, S. Liu, W. Liu, H. Ouyang, H. Li, Influence of quenchinduced precipitation on aging behavior of $\mathrm{Al}-\mathrm{Zn}-\mathrm{Mg}-\mathrm{Cu}$ alloy, Trans. Nonferrous Met. Soc. China 22 (2012) 1255-1263.

[33] Y. Deng, L. Wan, Y. Zhang, X. Zhang, Influence of Mg content on quench sensitivity of Al-Zn-Mg-Cu aluminium alloys, J. Alloys Compd. 509 (2011) 4636-4642.

[34] A.J. Bryant, The Effect of Composition upon the Quench-Sensitivity of Some Al-Zn-Mg Alloys, Journal of the Institute of Metals 94 (1966) 94-99. 

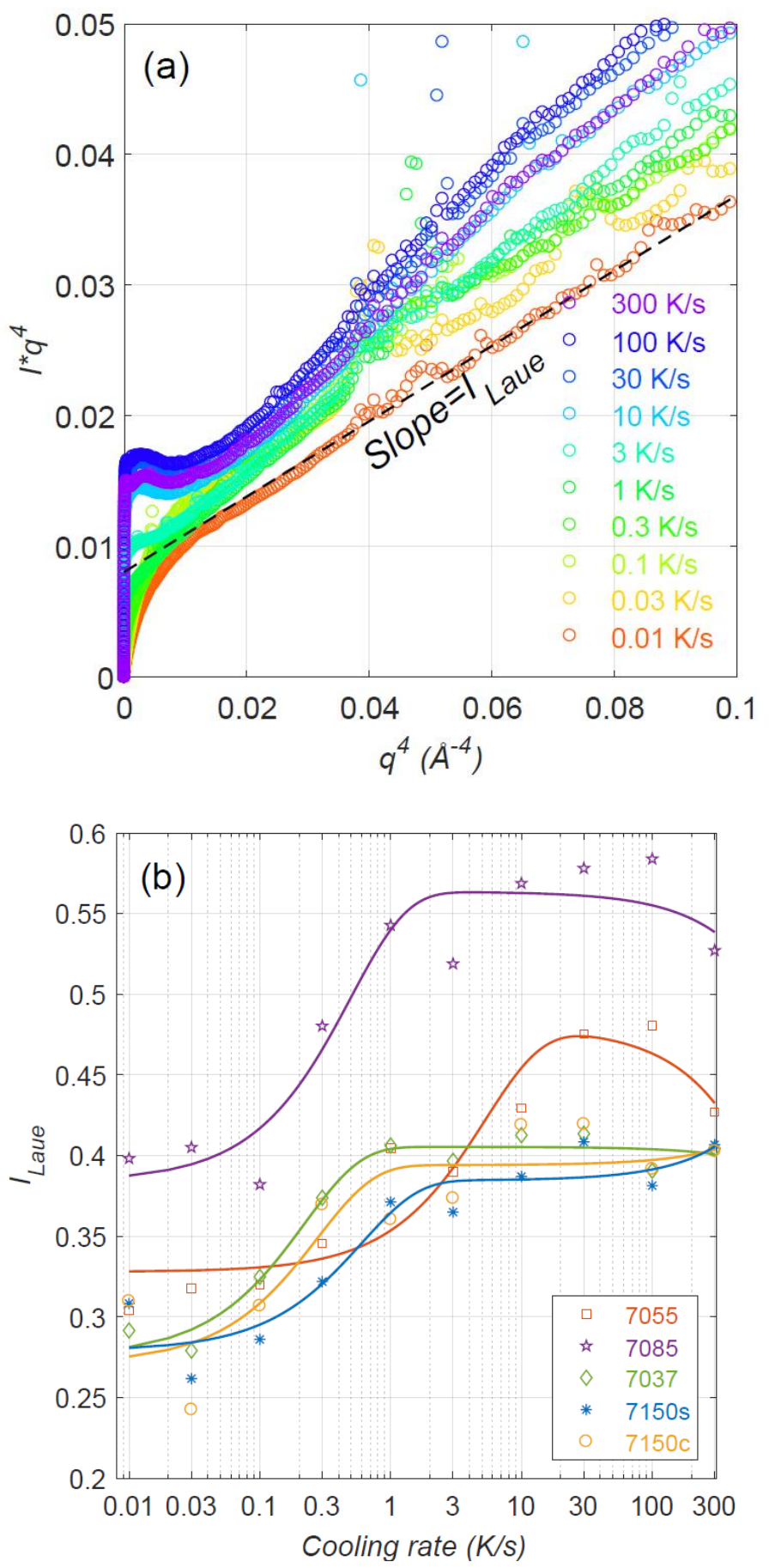

Figure 1: (a) $I_{\text {raw }} q^{4}$ vs. $q^{4}$ plot for alloy 7055 , the slope of which is equal to $I_{\text {Laue; }}$ (b) The Laue background changes with cooling rates for all studied alloys 
published as: Materials \& Design, 142, 2018, Pages 259-267

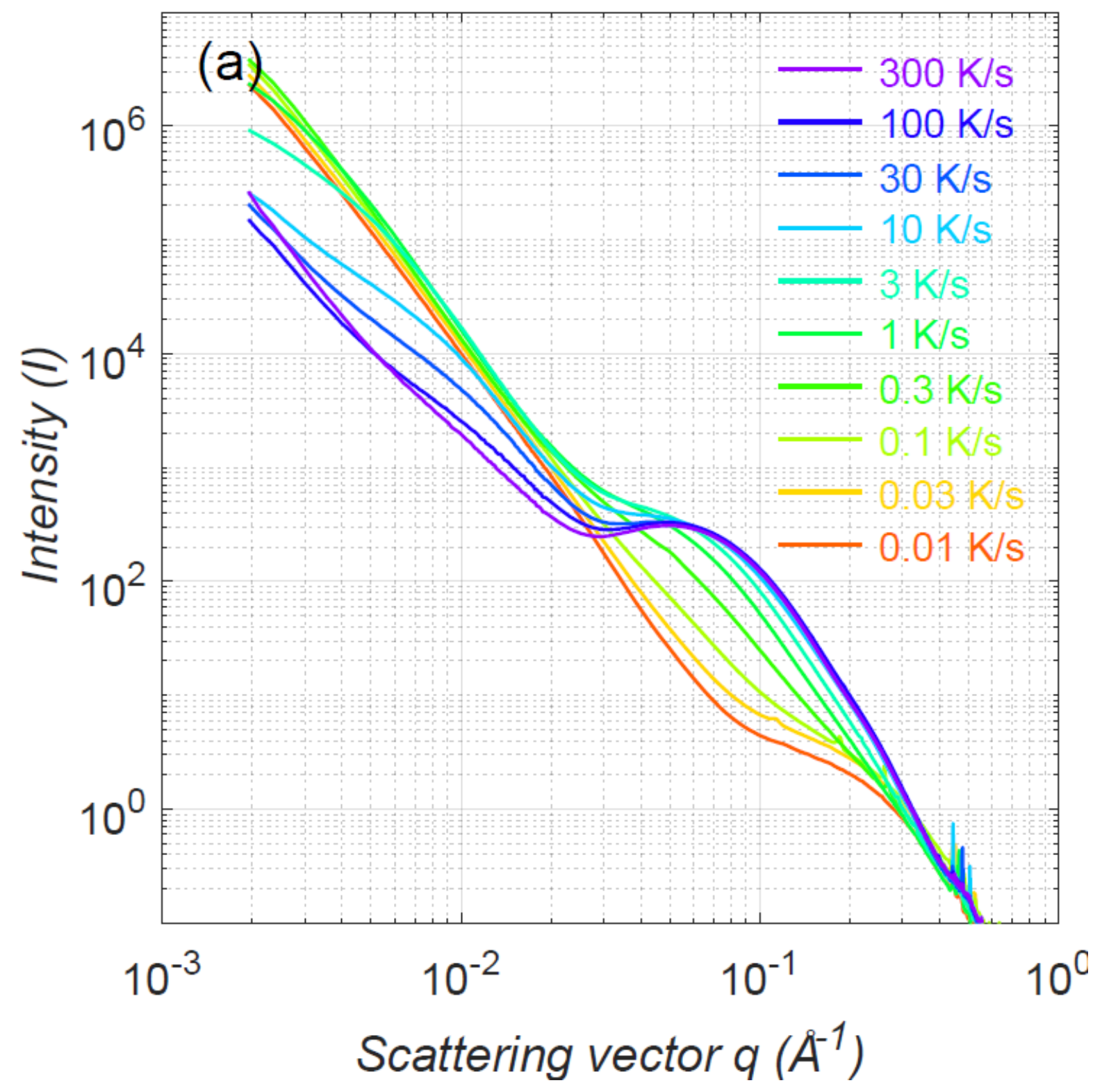


published as: Materials \& Design, 142, 2018, Pages 259-267

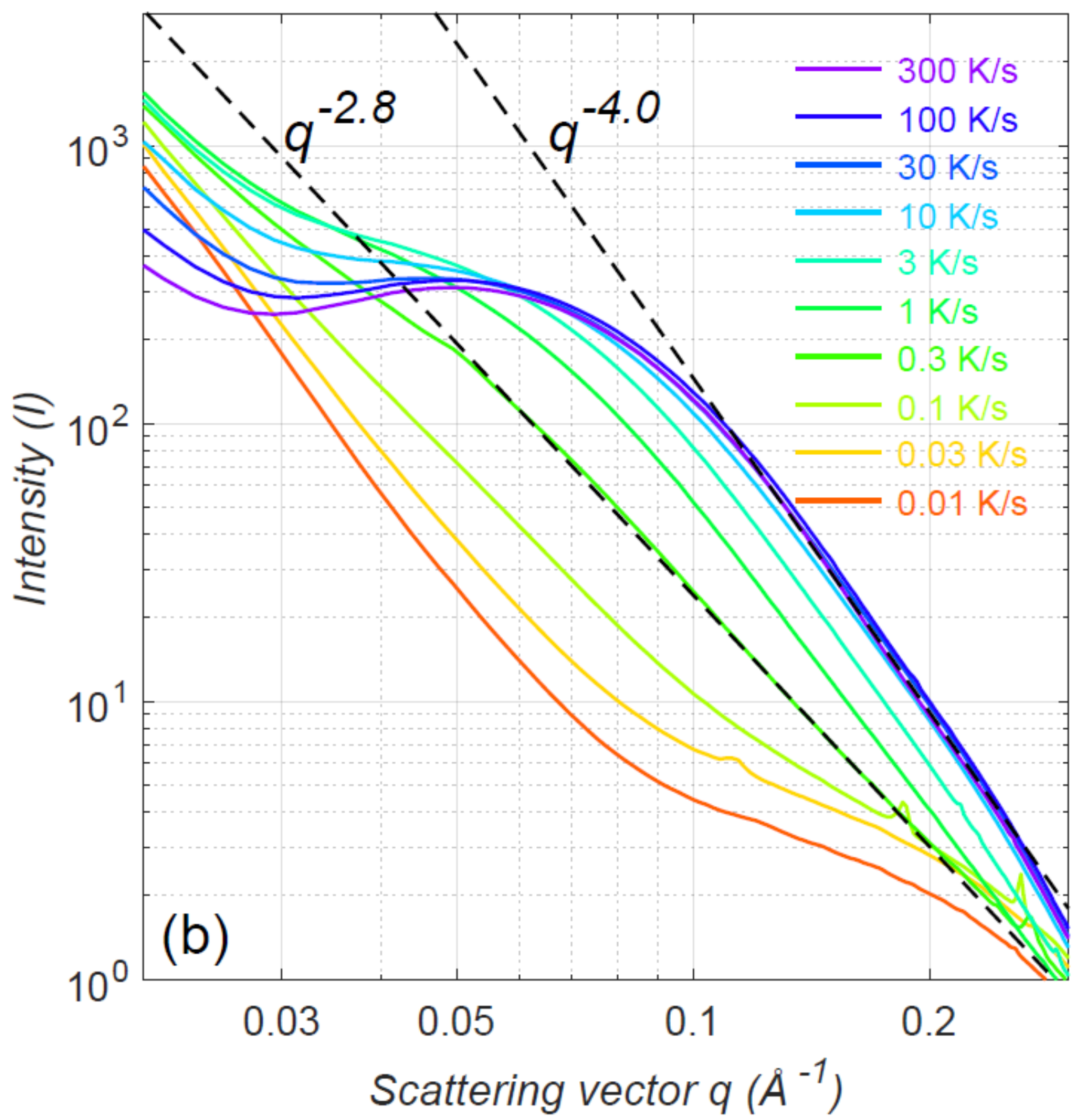




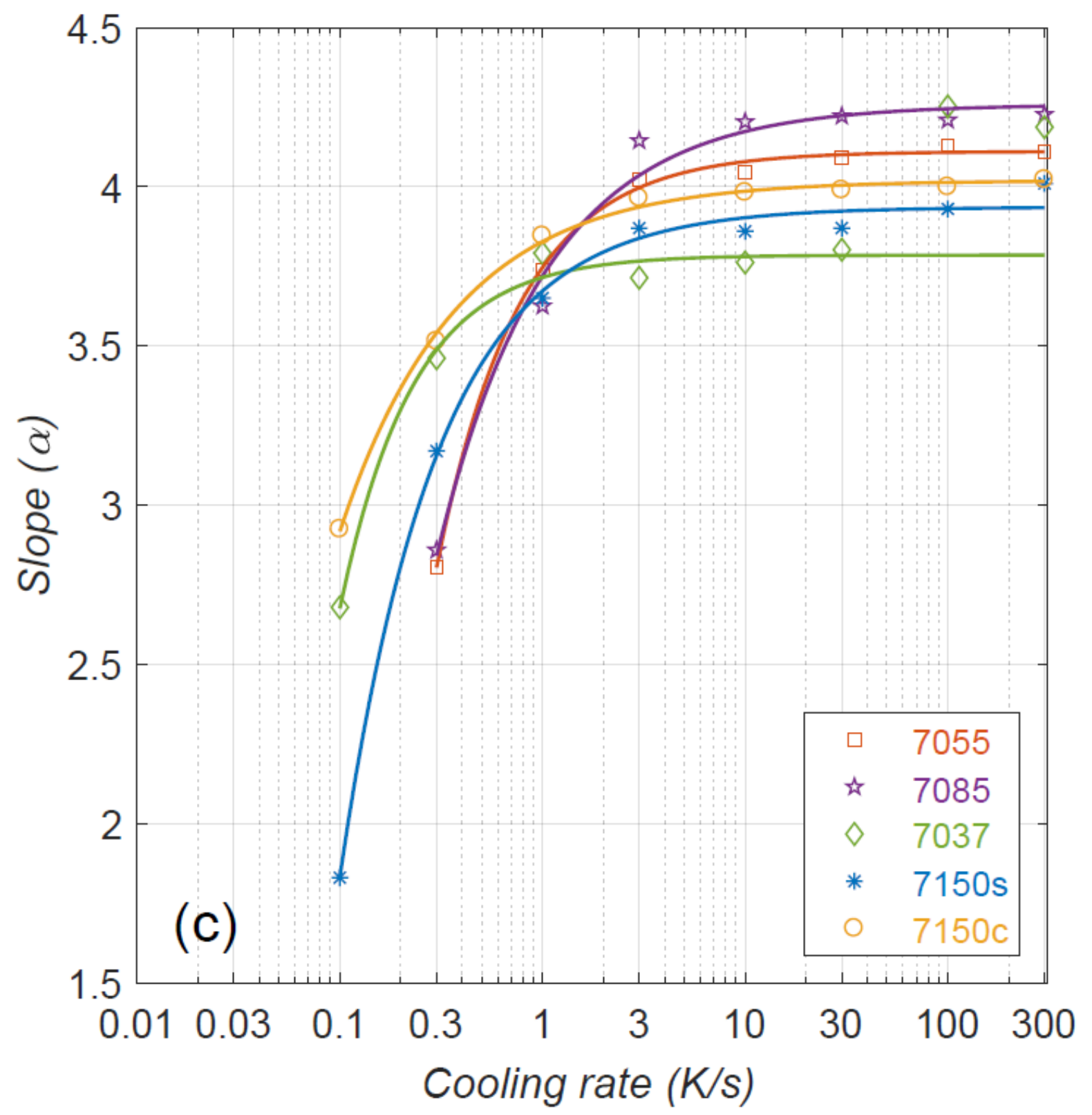

Figure 2: (a) Scattered intensity after Laue correction vs. $q$, (b) Plots showing how the $\alpha$ values changes with cooling rate for alloy 7055, (c) Plots showing how the $\alpha$ values changes with cooling rate for all studied alloys 

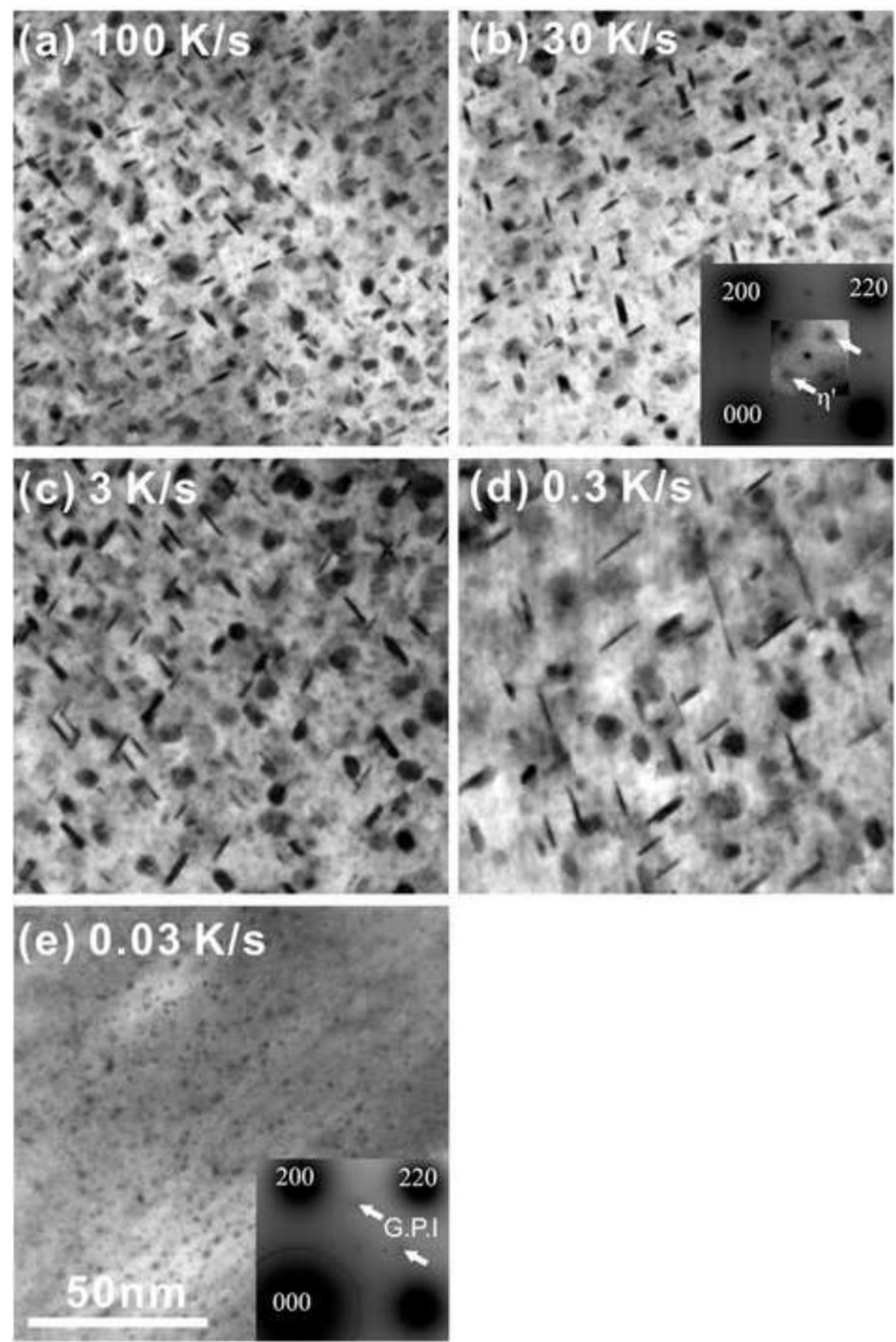

Figure 3: TEM images and SAD patterns of ageing induced precipitates in 7055-T6 for different cooling conditions. All images are taken from the $<110>$ Al direction. 
published as: Materials \& Design, 142, 2018, Pages 259-267

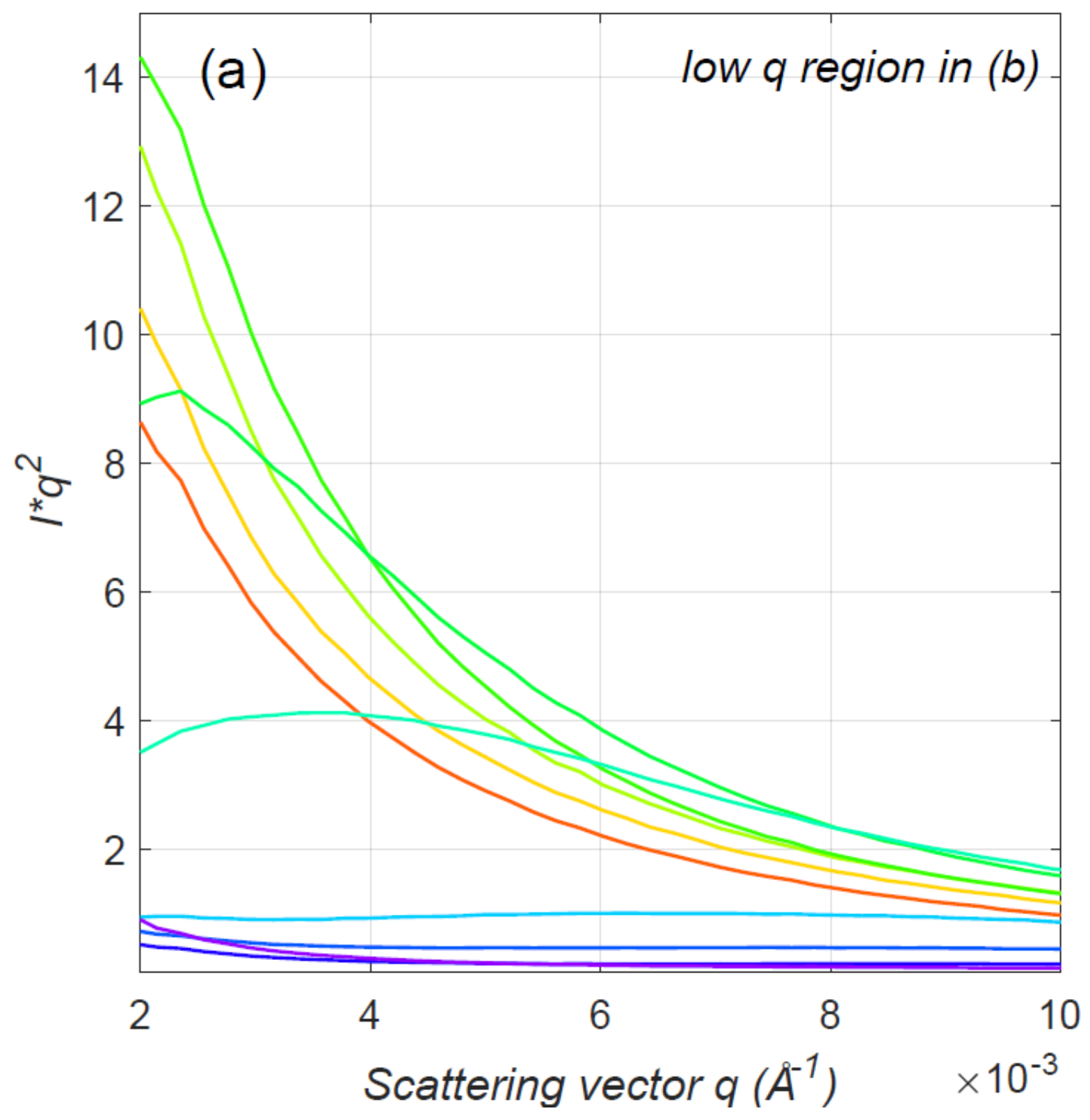


published as: Materials \& Design, 142, 2018, Pages 259-267

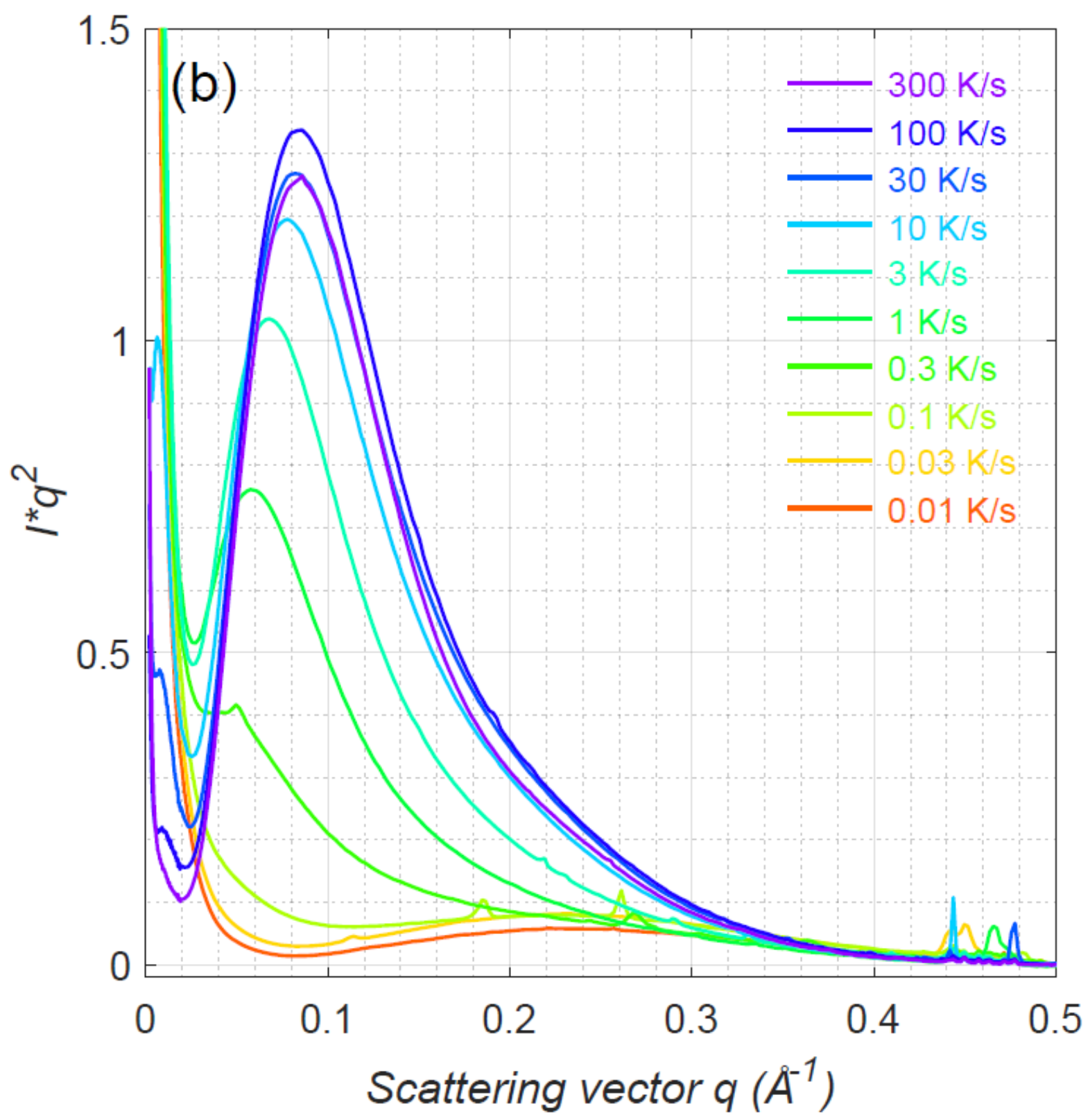




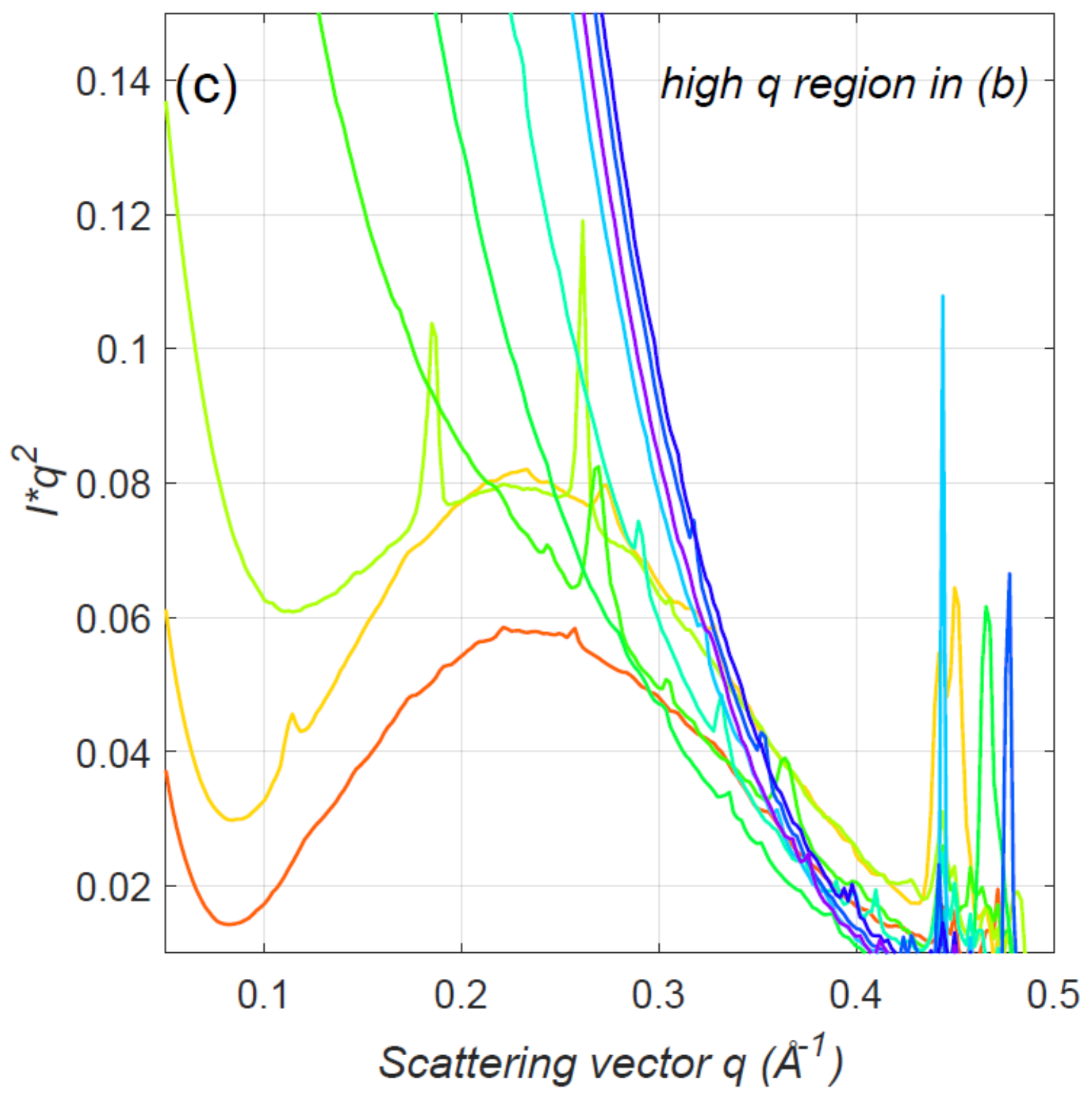

Figure 4: Kratky plot (I.q ${ }^{2}$ vs. q) for alloy 7055-T6 for the full range of cooling conditions shown for (a) low $q$ region, (b) full range of $q$ values, and (c) higher $q$ region. 


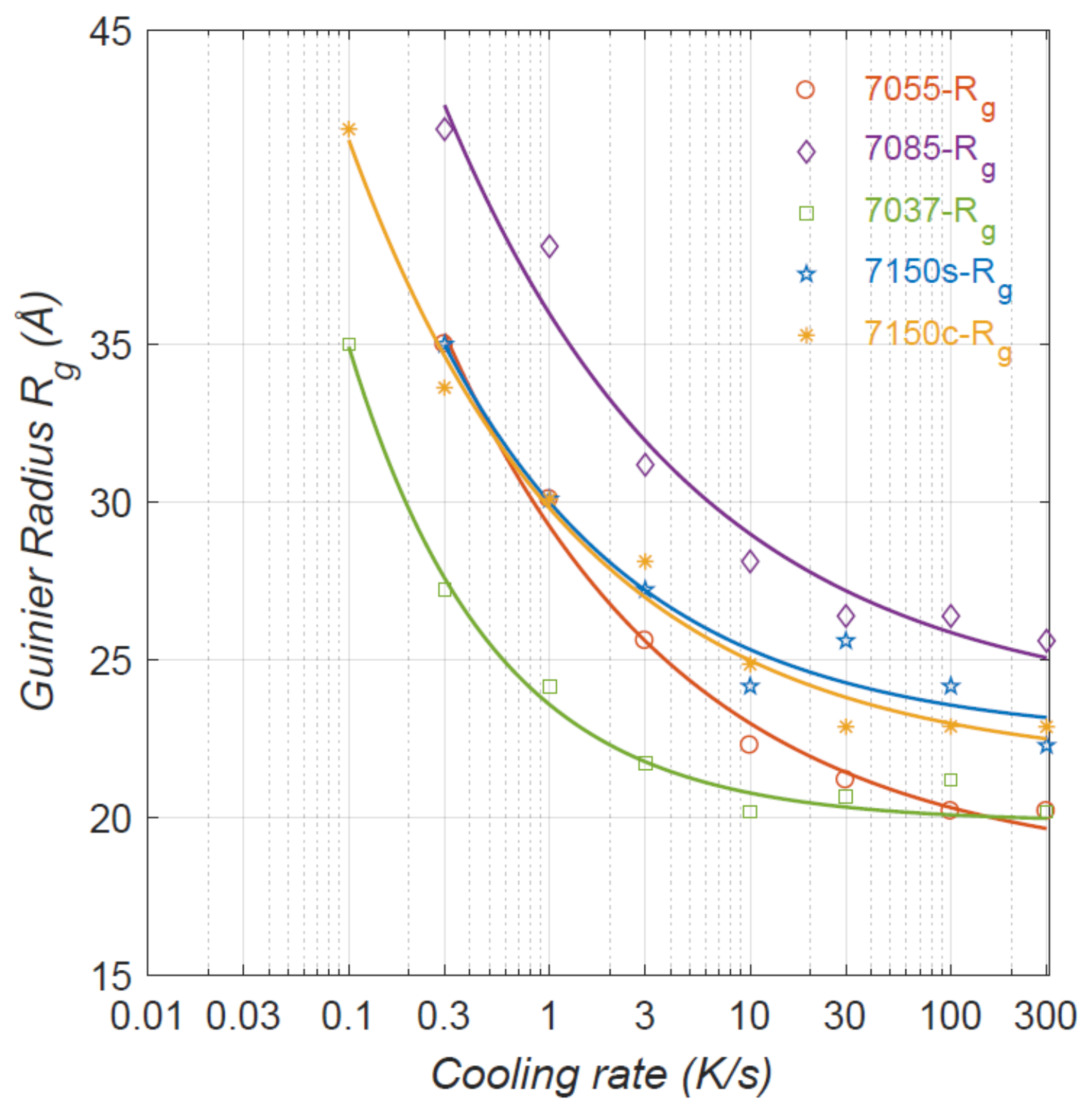

Figure 5: Guinier Radius $\boldsymbol{R}_{\mathrm{g}}$ of $\eta^{\prime}$ precipitates formed during ageing, plotted against cooling rate for all the studied alloys, The curves end at low cooling rates where there is no valid data for $\eta^{\prime}$ precipitates, demonstrating that no $\eta^{\prime}$ precipitates can be detected. 
published as: Materials \& Design, 142, 2018, Pages 259-267

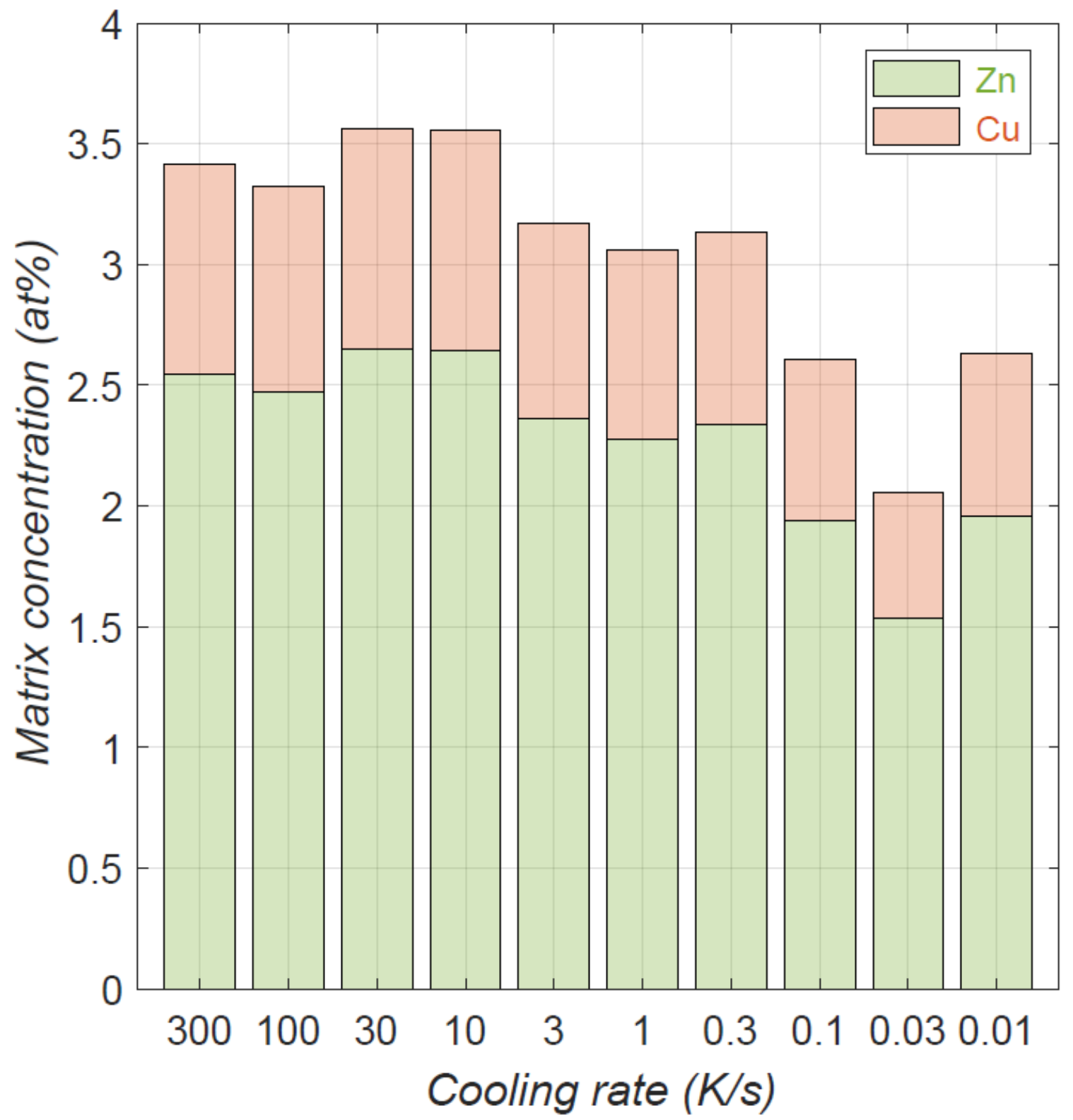

Figure 6: Calculated matrix concentration in the T6 condition for different cooling rates for alloy 7055 . 
published as: Materials \& Design, 142, 2018, Pages 259-267

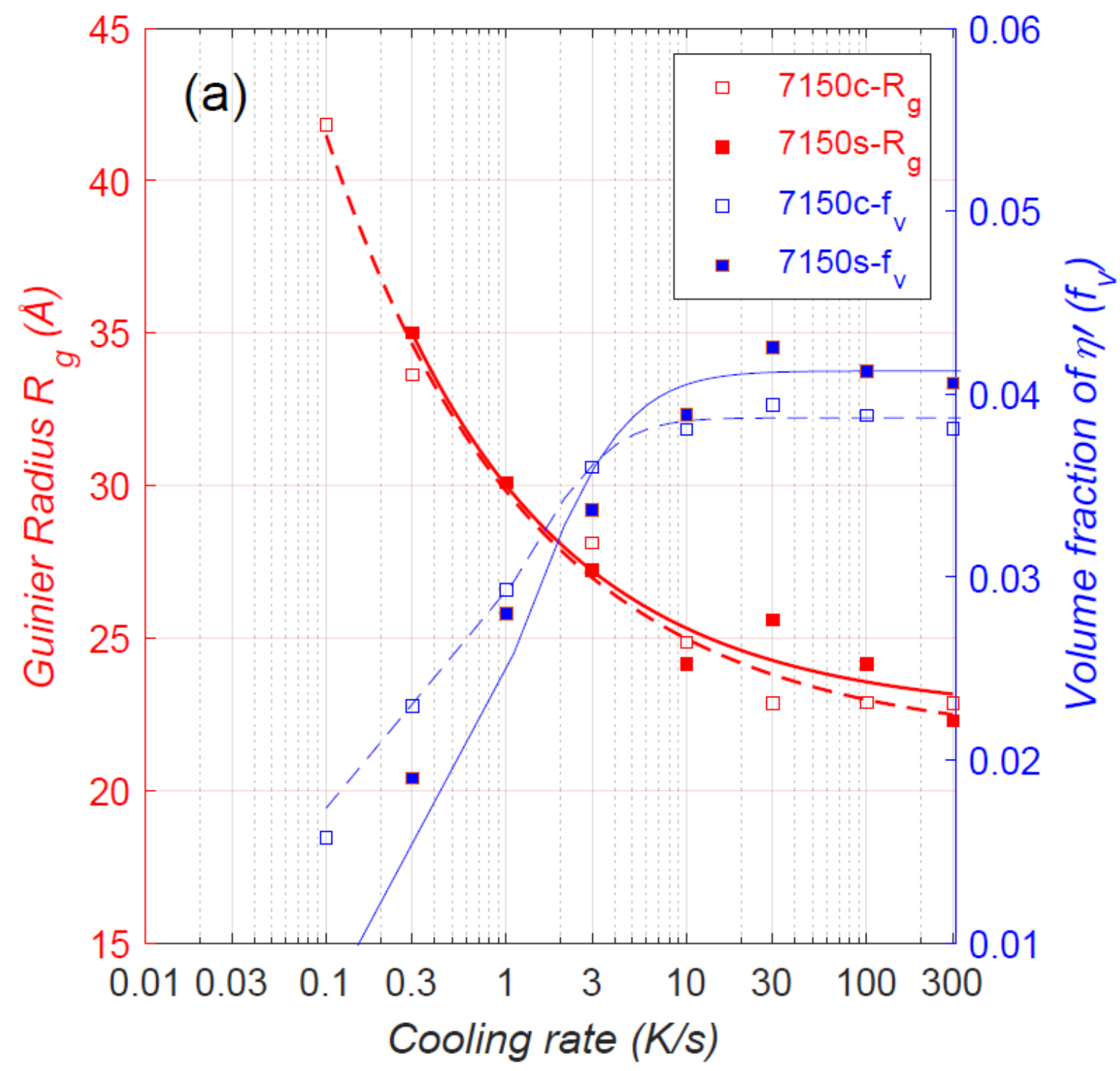


published as: Materials \& Design, 142, 2018, Pages 259-267

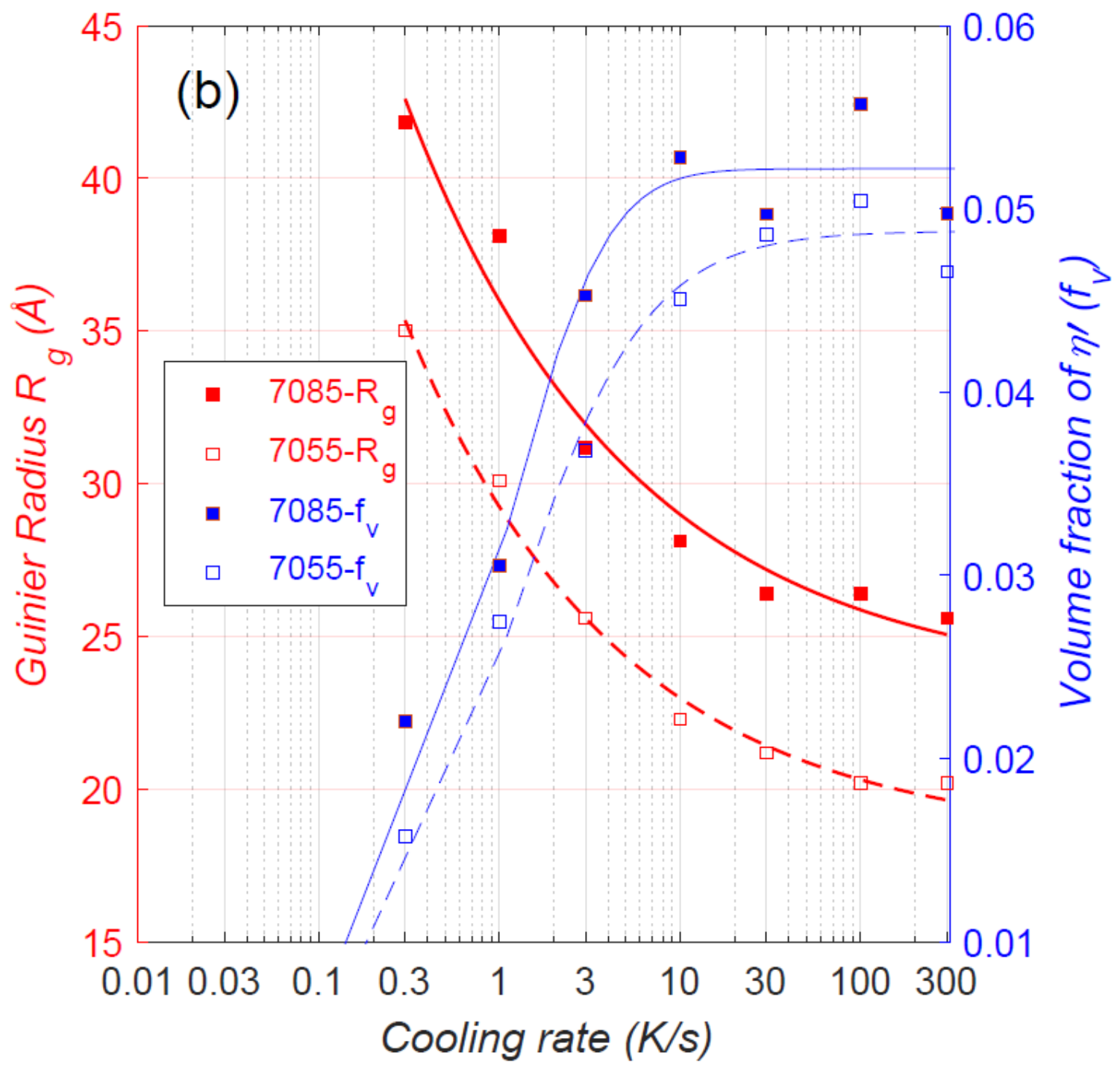




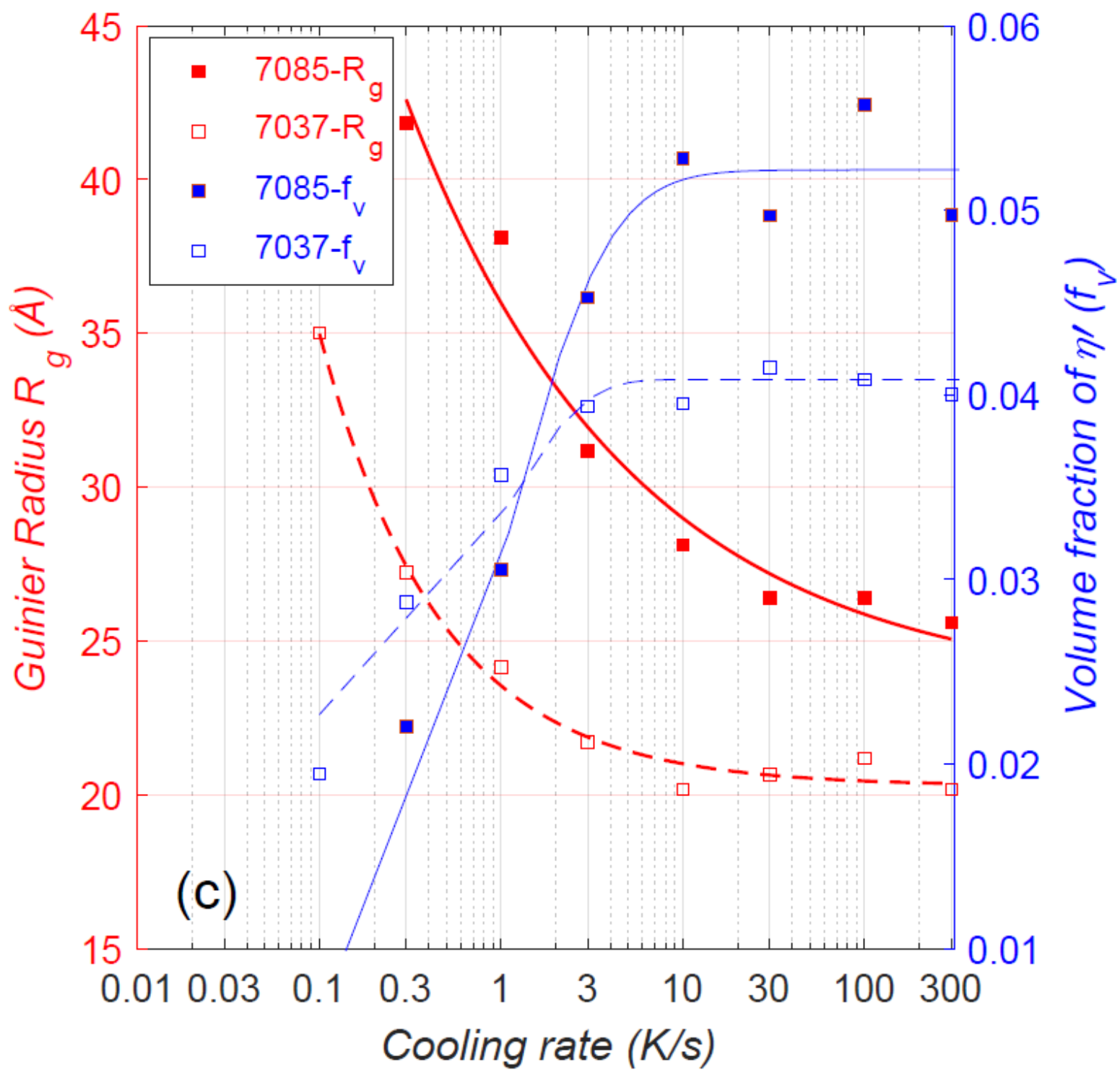

Figure 7: Volume fraction and Guinier Radius of $\eta$ ' precipitates in the T6 condition as a function of cooling rate for the studied alloys (a) $7150 \mathrm{C}$ and $7150 S$, (b) 7055 and 7085 and (c) 7085 and 7037. 